\title{
Successful Sequential Therapy Involving Regorafenib after Failure of Sorafenib in a Patient with Recurrent Hepatocellular Carcinoma after Liver Transplantation
}

\author{
Soon Kyu Lee', Jeong Won Jang', Heechul Nam¹, Pil Soo Sung², Si Hyun Bae², Jong Young Choi', Seung Kew Yoon \\ 'Division of Hepatology, Department of Internal Medicine, Seoul St. Mary's Hospital, Seoul; ${ }^{2}$ Division of Hepatology, Department of \\ Internal Medicine, Eunpyeong St. Mary's Hospital, College of Medicine, The Catholic University of Korea, Seoul, Korea
}

Received Jan. 7, 2020

Revised Feb. 18, 2020

Accepted Feb. 20, 2020
The efficacy and safety of sequential systemic therapy for the treatment of recurrent hepatocellular carcinoma (HCC) after liver transplantation (LT) are not well established. This study describes a successful experience where sequential therapy with sorafenib followed by regorafenib was used to treat recurrent HCC in a 54-year old male LT recipient. After HCC recurred in both lungs 10 months after $\mathrm{LT}$, sorafenib was administered with radiation therapy to treat pulmonary metastases. However, after 4 months of sorafenib treatment showed progressive pulmonary metastases, sequential regorafenib treatment was started. After 3 months (cycles) of regorafenib treatment, tumor response was partial, and after 6 months (cycles), disease status remained stable without signs of progression or drug-related serious adverse events. This case suggests that sequential systemic therapy is feasible in patient with recurrent HCC after LT. (J Liver Cancer 2020;20:84-89)

Keywords: Liver transplantation; Hepatocellular carcinoma; Sorafenib; Regorafenib; Metastasis

\section{INTRODUCTION}

Liver transplantation (LT) is the ultimate treatment modality for hepatocellular carcinoma (HCC) with a view of simultaneously curing HCC and liver cirrhosis. ${ }^{1}$ However, HCC recurrence after LT remains a concern. To reduce the recurrence rate and donor shortage-related problems, several criteria have been developed for selecting candidates for LT. ${ }^{1}$ The Milan criteria are the most frequently used and include a single tumor measuring $\leq 5 \mathrm{~cm}$ or 3 tumors each measuring

\footnotetext{
Corresponding author : Jeong Won Jang

Division of Hepatology, Department of Internal Medicine, Seoul St. Mary's Hospital, 222 Banpo-daero, Seocho-gu, Seoul 06591, Korea

Tel. +82-2-2258-2073, Fax. +82-2-599-3589

E-mail; garden@catholic.ac.kr

https://orcid.org/0000-0003-3255-8474
}

$\leq 3 \mathrm{~cm}^{2}$ Some believe that the Milan criteria are too strict; thus, the University of California San Francisco (UCSF) or Up-to-7 criteria were created to increase the options for choosing candidates for LT with reasonable disease-free survival. $^{3,4}$ In patients selected using these criteria, the 5-year disease-free survival rates have been reported as $64.1-92 \% .^{2-4}$

In patients with recurrent HCC, multidisciplinary management and diverse approaches according to the recurrence status are needed. ${ }^{5}$ However, some patients remain unmet by the treatment strategies. In patients with multifocal distant metastases after LT, the feasibility and safety of sequential systemic therapy have not been evaluated. This article reports a case study of a patient with recurrent HCC after LT, successfully treated with a sequential treatment of sorafenib followed by regorafenib. 


\section{CASE REPORT}

\section{Clinical findings}

A 54-year-old man visited our outpatient clinic with progressing pulmonary metastases of HCC. Fifteen months pri- or to his first visit, he had received living-donor LT (LDLT) because of his hepatitis B virus-related HCC. At the time of LDLT, his Child-Pugh score was 10 (class C) and his alphafetoprotein (AFP) level, $26.3 \mathrm{ng} / \mathrm{mL}$. The tumor was within the Milan Criteria, according to liver dynamic magnetic resonance imaging. However, pathologically, his tumors
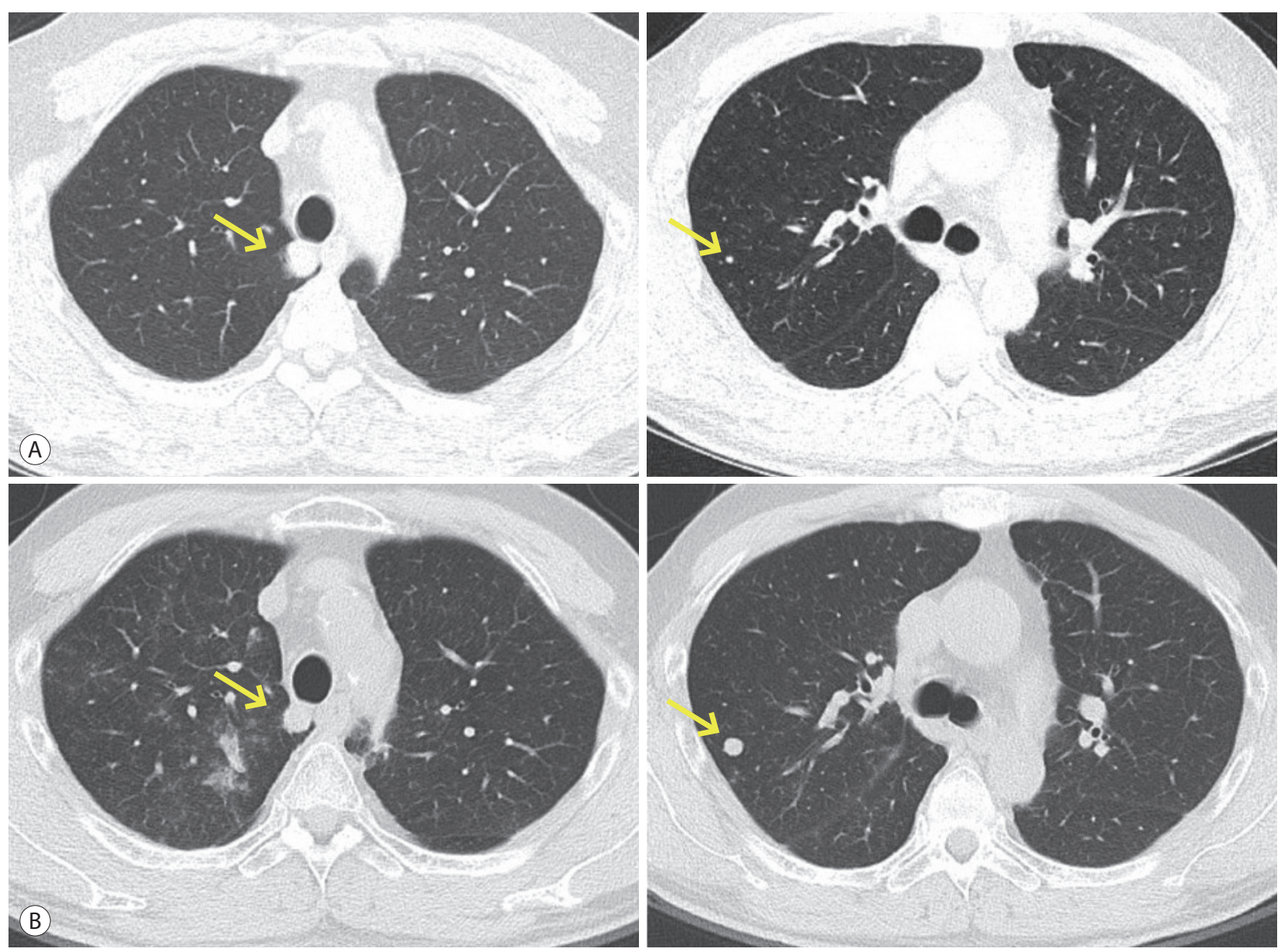

Figure 1. Initial chest computed tomography findings. (A) Pulmonary metastases (arrows) before sorafenib treatment. (B) Overall pulmonary metastases (arrows) progression after sorafenib treatment.

Table 1. Pulmonary metastases progress during sorafenib treatment

\begin{tabular}{lccccc}
\hline Image & Lesion $\mathbf{1}(\mathbf{m m})$ & Lesion $\mathbf{2}(\mathbf{m m})$ & Sum $^{*}(\mathbf{m m})$ & AFP $(\mathbf{n g} / \mathbf{m L})$ & PIVKA-II $(\mathbf{m A U} / \mathbf{m L})$ \\
\hline A & 14.2 & 3.5 & 14.2 & 1.6 & 164 \\
B & 13.6 & 10.5 & 24.1 & 1.9 & 292 \\
\hline
\end{tabular}

'A' is pulmonary metastases before sorafenib treatment (outside film). ' $B$ ' is pulmonary metastases after 4 months of sorafenib treatment. AFP, alpha-fetoprotein; PIVKA-II, protein induced by vitamin $\mathrm{K}$ absence or antagonist-II; mRECIST, modified response evaluation criteria in solid tumors.

"Sum of the size of lesion 1 and lesion 2. According to the mRECIST criteria, the sum of two target lesions per organ is calculated. The initial size of each target lesion should be more than $10 \mathrm{~mm}$. 
were more than five in number, with the largest being $3.5 \mathrm{~cm}$. The HCC had recurred 10 months after LDLT, appearing as pulmonary metastases without HCC recurrence in the transplanted liver. A combination of sorafenib, the firstline systemic therapy recommended in the guidelines, ${ }^{6-8}$ and radiation therapy (RT) on right upper lung lesion (total 60 gray with 30 fraction) was used for treating the pulmonary metastases. However, after 4 months of sorafenib treatment, the patient showed no response and the overall pulmonary metastases had progressed.
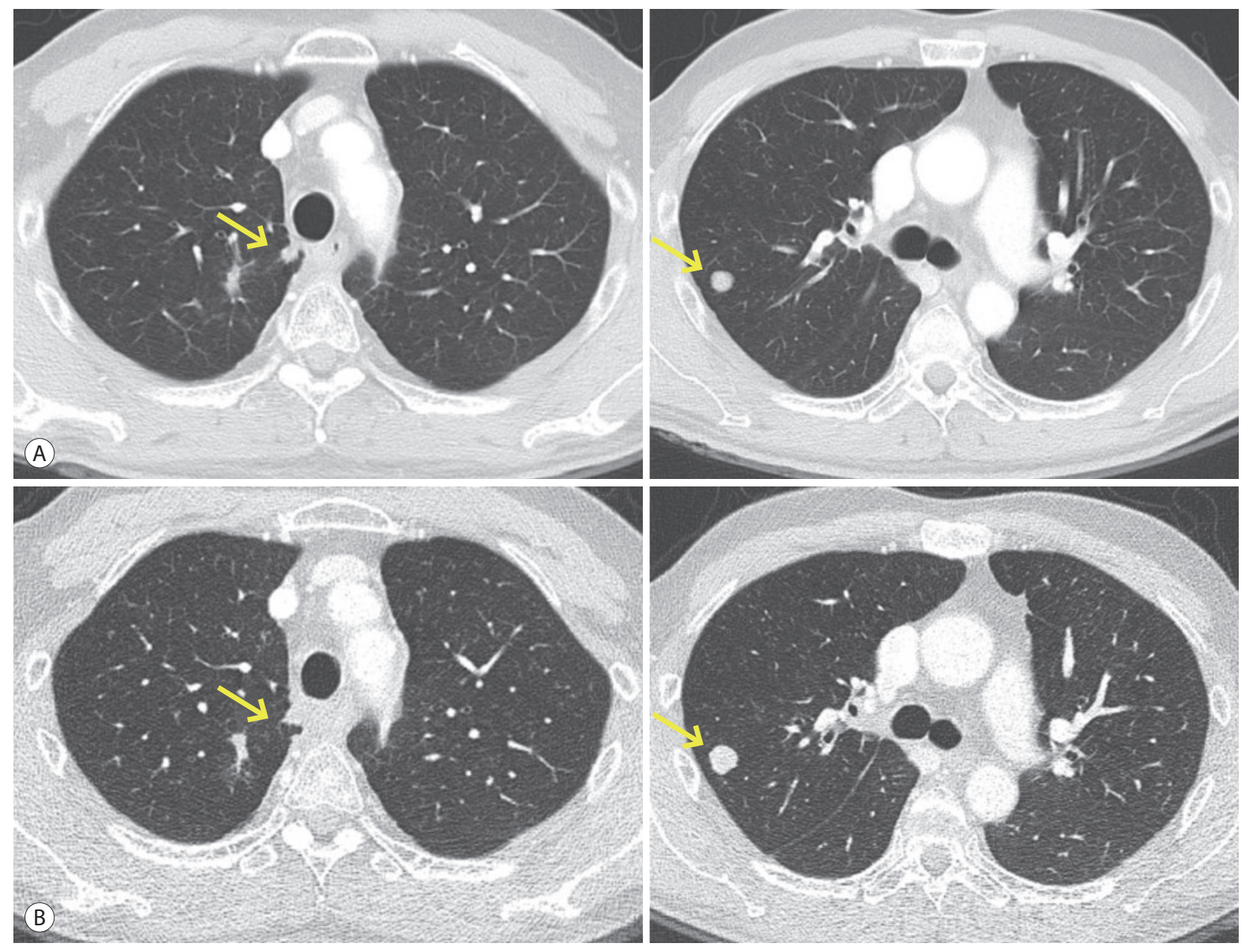

Figure 2. Follow-up chest computed tomography findings. (A) Overall partial response of pulmonary metastases (arrows) after 3 cycles of regorafenib. (B) Overall stable status of pulmonary metastases (arrows) after 6 cycles of regorafenib.

Table 2. Pulmonary metastases change during regorafenib treatment

\begin{tabular}{lccccccc}
\hline Image & $\begin{array}{c}\text { Lesion } \mathbf{1} \\
(\mathbf{m m})\end{array}$ & $\begin{array}{c}\text { Lesion } \mathbf{2} \\
(\mathbf{m m})\end{array}$ & $\begin{array}{c}\text { Sum }^{*} \\
(\mathbf{m m})\end{array}$ & $\begin{array}{c}\text { Calculation of response } \\
(\mathbf{\%})\end{array}$ & Response & $\begin{array}{c}\text { AFP } \\
(\mathbf{n g} / \mathbf{m L})\end{array}$ & $\begin{array}{c}\text { PIVKA-II } \\
(\mathbf{m A U} / \mathbf{m L})\end{array}$ \\
\hline A & 6.1 & 10.7 & 16.8 & $(16.8 / 24.1) \times 100=69.7$ & Partial response & 2.0 & 108 \\
B & 6.3 & 13.1 & 19.4 & $(19.8 / 16.8) \times 100=117.9$ & Stable disease & 4.3 & 130 \\
\hline
\end{tabular}

'A' is pulmonary metastases after 3 months (cycles) of regorafenib. ' $B$ ' is pulmonary metastases after 6 months (cycles) of regorafenib. AFP, alpha-fetoprotein; PIVKA-II, protein induced by vitamin K absence or antagonist-II; mRECIST, modified response evaluation criteria in solid tumors.

"Sum of the size of lesion 1 and lesion 2. According to the mRECIST criteria, the sum of two target lesions per organ is calculated. The initial size of each target lesion should be more than $10 \mathrm{~mm}$. 
When the patient visited our clinic with progressive disease after sorafenib treatment, physical examination showed no subjective symptoms or abnormalities. On the initial complete blood cell count check, his white blood cell count was $3.4 \times 10^{6} / \mathrm{L}$, hemoglobin concentration, $9.6 \mathrm{~g} / \mathrm{dL}$, and platelet count, $73 \times 10^{6} / \mathrm{L}$. His liver function was tolerable, with an albumin concentration of $3.7 \mathrm{~g} / \mathrm{dL}$, total bilirubin concentration of $0.67 \mathrm{mg} / \mathrm{dL}$, and an international normalized ratio of 1.03. Tumor marker concentrations were as follows: AFP, $1.6 \mathrm{ng} / \mathrm{mL}$; protein induced by vitamin $\mathrm{K}$ absence-II (PIVKA-II), $42 \mathrm{mAU} / \mathrm{mL}$.

\section{Image findings}

The initial 3-phase liver dynamic computed tomography (CT) revealed patent hepatic vessels and no clearly visible recurrence of HCC in the graft liver. The initial chest CT at our hospital showed an increase in the size and number of multiple nodules in both lungs (Fig. 1B). This finding suggested progressive pulmonary metastases compared with the images taken before sorafenib treatment, 10 months after LDLT, upon initial pulmonary metastases identification (Fig. 1A). After treatment with sorafenib and RT for 4 months, the pulmonary metastases had progressed in size and number (Fig. 1B, Table 1).

\section{Diagnosis and treatment progress}

The patient's clinical and radiological findings showed that he had recurrent HCC after LDLT and progressive disease involving pulmonary metastases even after sorafenib treatment. He had stage C Barcelona Clinic Liver Cancer (BCLC), and the stage of modified Union for International Cancer Control (mUICC) was IVB (T0 after LT, N0, M1). His liver function was preserved, as shown by a Child-Pugh total score of 5, and his performance score of Eastern Cooperative Oncology Group (ECOG) was also tolerable (score 0$).{ }^{9}$

We decided to prescribe regorafenib (160 mg qd for 3 weeks), followed by rest for 1 week. We designated this 4 -week-period as 1 cycle of treatment. During the regorafenib treatment period, the patient exhibited hand-foot syndrome (World Health Organization [WHO] grade II) and abdominal pain with diarrhea. After 3 cycles of regorafenib treatment, his first response was evaluated using the modified Response Evaluation Criteria in Solid Tumors (RECIST) version 1.1. We observed no newly developed metastatic nodules in either lung, and to some extent, a decrease in the size of metastatic nodules. His overall response was classified as a partial response (Fig. 2A, Table 2). Based on the response evaluation, we continued the regorafenib treatment using the same dose and 4-week cycle. After 6 cycles of regorafenib treatment, the pulmonary metastatic nodules remained but the disease was stable (Fig. 2B, Table 2). He has continued regorafenib treatment, and his disease remains in a relatively controlled state.

\section{DISCUSSION}

In this case report, we described a patient who showed a relatively good response to sequential therapy with regorafenib after disease progression, following sorafenib treatment. Interestingly, the patient was treated for recurrent HCC after LT. The patient had good liver function and tolerated the sequential systemic therapy. The disease has been stable for 6 months ( 6 treatment cycles) with continuous regorafenib treatment, still being administered to the patient.

Regorafenib is a multi-kinase inhibitor that inhibits the signaling involved in angiogenesis, metastasis, and the tumor microenvironment. ${ }^{10-12}$ It has been shown to significantly increase survival compared with placebo after failure of sorafenib treatment in HCC patients. ${ }^{13}$ One study on sequential treatment involving sorafenib followed by regorafenib reported a significantly longer time to disease progression, regardless of the sorafenib dose or pace of disease progression. ${ }^{14}$ However, the effects and safety of regorafenib have not been thoroughly evaluated in settings of LT. We found that this LT recipient exhibited an effective response to the sequential treatment with regorafenib after sorafenib failure. The patient had good liver function with the graft liver during treatment, which may have increased his tolerance of the regorafenib treatment.

Our patient had tolerable side effects such as hand-foot 
syndrome and diarrhea. Recently, a group from Europe and Argentina reported preliminary safety data from their study of regorafenib treatment after sorafenib failure in LT patients. ${ }^{15}$ As seen in our patient, the most common adverse symptoms were fatigue and dermatological reaction. Our patient did not experience rejection during treatment, also consistent with previous study results. These findings suggest that sequential regorafenib treatment might be both effective and safe for recurrent HCC treatment in selected patients, after LT.

Recently, several new systemic drugs, including regorafenib, nivolumab, and cabozantinib, have been introduced as second-line options after sorafenib failure. ${ }^{16,17}$ Some studies have demonstrated that after LT, patients with high AFP concentration $(>200 \mathrm{ng} / \mathrm{mL})$, large tumor size $(>3 \mathrm{~cm})$, and high neutrophil-lymphocyte ratio $(\geq 5)$ might be at risk of HCC recurrence. ${ }^{18}$ The treatment strategy is of importance for prognosis improvement in HCC recurrence after LT, and further studies on the effectiveness and safety of sequential therapy after sorafenib failure are needed. Our patient showed good response to sequential therapy, indicating the need for further studies on the optimal treatment strategy for recurrent HCC after LT.

Summarily, we reported on a successful case of sequential therapy for recurrent HCC after LT. Regorafenib treatment after disease progression following sorafenib produced a reasonable response in this patient. However, decision-making for the treatment of recurrent HCC after LT remains a difficult problem for clinicians. The outcome of this case suggests that as with non-LT patients, sequential treatment of HCC with regorafenib after sorafenib may be feasible without compromising graft stability in post-LT patients with progressive disease.

\section{ACKNOWLEDGMENTS}

This case report was approved by the Institutional Review Board of Seoul St. Mary's Hospital (KC20ZISI0025).

\section{Conflicts of Interest}

The authors declare no competing interests.

\section{REFERENCES}

1. Sapisochin G, Bruix J. Liver transplantation for hepatocellular carcinoma: outcomes and novel surgical approaches. Nat Rev Gastroenterol Hepatol 2017;14:203-217.

2. Mazzaferro V, Regalia E, Doci R, Andreola S, Pulvirenti A, Bozzetti F, et al. Liver transplantation for the treatment of small hepatocellular carcinomas in patients with cirrhosis. N Engl J Med 1996;334:693699.

3. Yao FY, Xiao L, Bass NM, Kerlan R, Ascher NL, Roberts JP. Liver transplantation for hepatocellular carcinoma: validation of the UCSF-expanded criteria based on preoperative imaging. Am J Transplant 2007;7:2587-2596.

4. Mazzaferro V, Llovet JM, Miceli R, Bhoori S, Schiavo M, Mariani L, et al. Predicting survival after liver transplantation in patients with hepatocellular carcinoma beyond the Milan criteria: a retrospective, exploratory analysis. Lancet Oncol 2009;10:35-43.

5. Kneuertz PJ, Cosgrove DP, Cameron AM, Kamel IR, Geschwind $J F$, Herman JM, et al. Multidisciplinary management of recurrent hepatocellular carcinoma following liver transplantation. J Gastrointest Surg 2012;16:874-881.

6. European Association for the Study of the Liver. EASL Clinical Practice Guidelines: management of hepatocellular carcinoma. J Hepatol 2018;69:182-236.

7. Korean Liver Cancer Association; National Cancer Center. 2018 Korean Liver Cancer Association-National Cancer Center Korea Practice Guidelines for the Management of Hepatocellular Carcinoma. Gut Liver 2019;13:227-299.

8. Heimbach JK, Kulik LM, Finn RS, Sirlin CB, Abecassis MM, Roberts $L R$, et al. AASLD guidelines for the treatment of hepatocellular carcinoma. Hepatology 2018;67:358-380.

9. Oken MM, Creech RH, Tormey DC, Horton J, Davis TE, McFadden $E T$, et al. Toxicity and response criteria of the Eastern Cooperative Oncology Group. Am J Clin Oncol 1982;5:649-655.

10. Wilhelm SM, Dumas J, Adnane L, Lynch M, Carter CA, Schutz G, et al. Regorafenib (BAY 73-4506): a new oral multikinase inhibitor of angiogenic, stromal and oncogenic receptor tyrosine kinases with potent preclinical antitumor activity. Int J Cancer 2011;129:245255.

11. Abou-Elkacem L, Arns S, Brix G, Gremse F, Zopf D, Kiessling F, et al. Regorafenib inhibits growth, angiogenesis, and metastasis in a highly aggressive, orthotopic colon cancer model. Mol Cancer Ther 2013;12:1322-1331.

12. Wilhelm SM, Carter C, Tang L, Wilkie D, McNabola A, Rong H, et al. BAY 43-9006 exhibits broad spectrum oral antitumor activity and targets the RAF/MEK/ERK pathway and receptor tyrosine kinases involved in tumor progression and angiogenesis. Cancer Res 2004;64:7099-7109. 
13. Bruix J, Qin S, Merle P, Granito A, Huang YH, Bodoky G, et al. Regorafenib for patients with hepatocellular carcinoma who progressed on sorafenib treatment (RESORCE): a randomised, doubleblind, placebo-controlled, phase 3 trial. Lancet 2017;389:56-66.

14. Finn RS, Merle P, Granito A, Huang YH, Bodoky G, Pracht M, et al. Outcomes of sequential treatment with sorafenib followed by regorafenib for HCC: additional analyses from the phase III RESORCE trial. J Hepatol 2018;69:353-358.

15. lavarone M, Invernizzi F, Czauderna C, Sanduzzi-Zamparelli M, Bhoori $S$, Amaddeo $G$, et al. Preliminary experience on safety of regorafenib after sorafenib failure in recurrent hepatocellular car- cinoma after liver transplantation. Am J Transplant 2019;19:31763184.

16. Rimassa L, Pressiani T, Merle P. Systemic treatment options in hepatocellular carcinoma. Liver Cancer 2019;8:427-446.

17. Vogel A, Saborowski A. Current strategies for the treatment of intermediate and advanced hepatocellular carcinoma. Cancer Treat Rev 2019;82:101946.

18. Halazun KJ, Najjar M, Abdelmessih RM, Samstein B, Griesemer $A D$, Guarrera JV, et al. Recurrence after liver transplantation for hepatocellular carcinoma: a new MORAL to the story. Ann Surg 2017;265:557-564. 